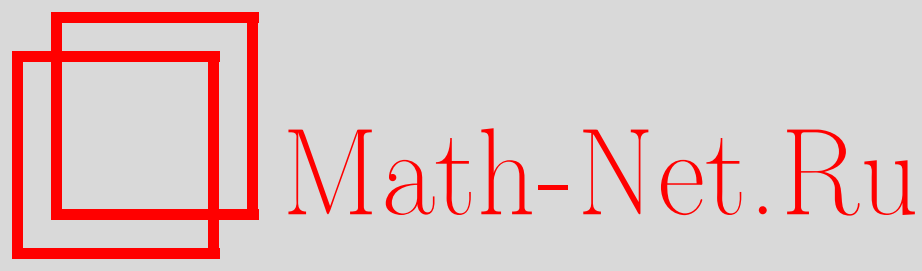

А. В. Акишин, О группах четного порядкас автоморфизмами, порождающими рекуррентные последовательности максимального периода, Дискрет. матем., 2014, том 26, выпуск 4, 15-22

DOI: https://doi.org/10.4213/dm1300

Использование Общероссийского математического портала Math-Net.Ru подразумевает, что вы прочитали и согласны с пользовательским соглашением http: //www . mathnet.ru/rus/agreement

Параметры загрузки:

IP: 3.93 .64 .190

26 апреля 2023 г., 15:20:59

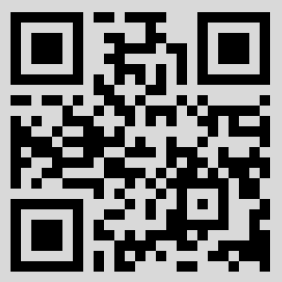




\title{
О группах четного порядка \\ с автоморфизмами, порождающими рекуррентные последовательности максимального периода
}

\author{
() 2014 г. А. В. Акишин*
}

\begin{abstract}
Пусть $G$ - конечная группа, $f$ - автоморфизм группы $G$. Тогда автоморфизм $f$ задает рекуррентную последовательность $\left\{a_{i}\right\}_{0}^{\infty}$ на группе $G$, уравнением $a_{i+1}=f\left(a_{i}\right)$. Если $a_{0}-$ начальный элемент этой последовательности, то ее период не превышает числа элементов порядка, равного порядку $a_{0}$. Таким образом, определенный интерес вызывает вопрос о существовании групп, у которых такая последовательность при некотором автоморфизме имеет максимально возможный период для любого начального состояния.

В статье продолжаются исследования групп, допускающих автоморфизмы максимального периода. Ранее был рассмотрен случай групп нечетного порядка. Оказалось, что такие группы необходимо являются абелевыми, и описана их структура. Здесь рассматриваются группы четного порядка и заканчивается описание конечных групп, допускающих автоморфизмы максимального периода.
\end{abstract}

Ключевые слова: группы, автоморфизмы, рекуррентные последовательности.

\section{1. Введение}

Изучение автоморфизмов групп и зависимости свойств группы от свойств группы ее автоморфизмов являются важными задачами в связи с широким применением автоморфизмов в различных прикладных вопросах, в частности, в криптографии. Одним из широко известных применений автоморфизмов в криптографических приложениях являются регистры сдвига с линейной обратной связью $[6,5,3,4,8]$. В настоящее время регистры сдвига с линейной обратной связью в том или ином виде используются как составные части поточных алгоритмов шифрования $[9,10,11]$.

В общем виде регистр сдвига с $n$ ячейками и линейной обратной связью над полем $F$ можно представить как линейное преобразование $A$ векторного пространства $V$ над полем $F$ размерности $n$. При этом для приложений наиболее важным является случай, когда линейное преобразование $A$ обратимо, то есть является автоморфизмом векторного пространства $V$. Однако пространство $V$ можно рассматривать и как абелеву группу, изоморфную прямому произведению $n$ экземпляров аддитивных

* Место работы: Московский институт радиотехники, электроники и автоматики, e-mail: sd45ki91@yandex.ru 
групп поля $F$. В этом случае линейное преобразование $A$ является автоморфизмом этой группы.

В приложениях регистры сдвига применяются, как правило, в качестве генераторов исходной гаммы, которая затем подвергается усложнению. В качестве примеров можно привести генератор Геффе [12], генератор Дженнингса [14], старт-стопный [13], пороговый и другие генераторы. При этом основным требованием к регистру сдвига является обеспечение большого периода вырабатываемой им последовательности.

В связи с этим уместно поставить вопрос о существовании групп с автоморфизмами максимального периода, то есть автоморфизмами, у которых все элементы группы одного порядка лежат на одном цикле. Отметим, что такие автоморфизмы являются регулярными (то есть не имеют неподвижных точек, за исключением единичного элемента) и также представляют самостоятельный интерес [2, 7]. Этот вопрос был поставлен в работе [1], где было получено полное описание конечных абелевых групп с автоморфизмами максимального периода и показано, что все конечные группы нечетного порядка с автоморфизмами максимального периода являются абелевыми. В настоящей работе продолжаются исследования групп с автоморфизмами максимального периода и рассматриваются конечные группы четного порядка.

\section{2. О строении групп четного порядка}

Сначала напомним некоторые определения.

Пусть $G$ - конечная группа. Символом $|a|$ будем обозначать порядок элемента $a \in G$, а через $C_{n}$ обозначим циклическую группу порядка $n$.

Автоморфизм $f$ группы $G$ назовем автоморфизмом максималъного периода, если для любых двух таких элементов $a, b \in G$, что $|a|=|b|$, существует целое число $n$, для которого

$$
f^{n}(a)=b \text {. }
$$

Иначе говоря, если рассматривать автоморфизм как подстановку на элементах группы, то у автоморфизма максимального периода все элементы одного порядка лежат на одном его цикле.

Докажем сначала следующее утверждение.

Лемма 1. Пусть $G$ - конечная 2-группа, обладающая автоморфизмами максимального периода. Тогда группа $G$ абелева.

Доказательство. Пусть $f$ - автоморфизм максимального периода группы $G, a$ - элемент максимального порядка. Если $|a|=2$, то для любого элемента $x \in G$ выполняется равенство $x^{2}=e$, откуда следует, что группа $G$ является элементарной абелевой группой. Рассмотрим случай, когда $|a|>2$.

Сначала заметим, что если $x \in G$ - произвольный элемент, то найдется такой элемент $b \in G$, что $x=b^{2^{k}}$ для некоторого $k \in \mathbb{Z}$, и $|b|=|a|$. Отсюда следует, что для доказательства леммы достаточно показать, что любые два элемента максимального порядка группы $G$ коммутируют.

Итак, пусть $b$ - еще один элемент максимального порядка. Покажем сначала, что $a^{2} b=b a^{2}$. Действительно, пусть $n$ - такое целое число, что $f^{n}(a)=a^{-1}$. Так как $|a|>2$, то $a^{-1} \neq a$. Тогда, так как $|b|=|a|$ и $\left|a b a^{-1}\right|=|a|$, то $f^{n}(b)=b^{-1}$ и 
$f^{n}\left(a b a^{-1}\right)=\left(a b a^{-1}\right)^{-1}$. Имеем

$$
a b a^{-1}=\left(a b^{-1} a^{-1}\right)^{-1}=f^{n}\left(a b^{-1} a^{-1}\right)=f^{n}(a) f^{n}\left(b^{-1}\right) f^{n}\left(a^{-1}\right)=a^{-1} b a,
$$

значит, $a^{2} b=b a^{2}$. В соответствии со сделанным выше замечанием отсюда следует, что элемент $a^{2}$ коммутирует со всеми элементами максимального порядка группы $G$, а следовательно, и со всеми элементами группы $G$. Иными словами, $a^{2} \in Z(G)$ - элемент центра. Более того, если $c \in G$ и $|c|<|a|$, то $c \in Z(G)$.

Рассмотрим теперь элемент $a b$. Возможны два варианта:

1) $|a b|<|a|$,

2) $|a b|=|a|$.

В первом случае элемент $a b$ лежит в центре группы $G$, и тогда

$$
a b=a^{-1}(a b) a=b a .
$$

Во втором случае из равенства

$$
b a^{-1}=\left(a b^{-1}\right)^{-1}=f^{n}\left(a b^{-1}\right)=f^{n}(a) f^{n}\left(b^{-1}\right)=a^{-1} b
$$

следует, что $a b=b a$. Это завершает доказательство леммы.

В работе [1] были доказаны следующие утверждения.

Теорема 1. Пусть $G$ - конечная группа четного порядка, $f$ - автоморфизм группы $G$ максимального периода. Тогда множество $H$ всех элементов нечетного порядка группы $G$ является абелевой нормальной подгруппой, допустимой относительно автоморфизма $f$.

Теорема 2. Конечная абелева р-группа $G$ обладает автоморфизмами максимального периода тогда и только тогда, когда она изоморфна одной из следующих групп:

1) $C_{p}^{m}$

2) $C_{p^{m}}, p \neq 2$,

3) $C_{4}$.

Следствие 1. Порядок группь четного порядка, обладающей автоморфизмом максимального периода, равен $2^{n} p^{m}$, где $p>2$ - простое число, $n$ u $m$ - некоторые натуральные числа. При этом ее силовская р-подгруппа нормальна.

Используя теорему 1 и следствие, из нее докажем следующее утверждение.

Теорема 3. Пусть $G$ - группа порядка $2^{n} p^{m}$, где $p>2$ - простое число, обладающая автоморфизмом $f$ максимального периода. Тогда возможны только следующие случаи :

1) $G \simeq C_{2}^{n} \ltimes C_{p}^{m}$,

2) $G \simeq C_{2}^{n} \ltimes C_{p^{m}}$,

3) $G \simeq C_{4} \ltimes C_{p}^{m}$,

4) $G \simeq C_{4} \ltimes C_{p^{m}}$.

Доказательство. В самом деле, в соответствии с теоремой 1 силовская $p$-подгруппа $H$ является абелевой нормальной подгруппой, допустимой относительно автоморфизма $f$. Следовательно, как показано в [1], для $H$ возможны только два случая: $H \simeq C_{p}^{m}$ и $H \simeq C_{p^{m}}$.

\footnotetext{
13десь значком $\ltimes$ обозначается полупрямое произведение групп.
} 
Далее, если через $K$ обозначить силовскую 2-подгруппу группы $G$, то из условий $K \cap H=\{e\}$ и $K H=G$ следует, что $G \simeq K \ltimes H$. Таким образом для завершения доказательства достаточно получить описание подгруппы $K$.

Рассмотрим факторгруппу $G / H$ и определим на ней отображение $\varphi$ правилом

$$
\varphi(x H)=f(x) H .
$$

То, что отображение $\varphi$ является автоморфизмом, очевидно. Так как $G / H \simeq K$, то, с учетом результатов работы [1] и леммы 1 , для доказательства теоремы достаточно показать, что $\varphi$ является автоморфизмом максимального периода на $G / H$.

Пусть $x H$ и $y H$ - два элемента из $G / H$ одного порядка $2^{k}$ для некоторого натурального $k$. Тогда

$$
x^{2^{k}} \in H \text { и } y^{2^{k}} \in H,
$$

т. е. $|x|=2^{k} p^{s}$ и $|y|=2^{k} p^{t}$ для некоторых целых $s, t \geqslant 0$. Пусть $x^{2^{k}}=a$ и $y^{2^{k}}=b$, где $a, b \in H$. Так как порядки элементов $a$ и $b$ являются степенями простого числа $p$, то существуют такие $a_{1} \in\langle a\rangle$ и $b_{1} \in\langle b\rangle$, что $a_{1}^{2^{k}}=a$ и $b_{1}^{2^{k}}=b$. Отметим, что при этом выполняются равенства

$$
a_{1} x=x a_{1} \text { и } b_{1} y=y b_{1} .
$$

Так как $a_{1}, b_{1} \in H$, то $x a_{1}^{-1} \in x H$ и $y b_{1}^{-1} \in y H$. Кроме того,

$$
\left(x a_{1}\right)^{2^{k}}=x^{2^{k}} a_{1}^{2^{k}}=a^{2^{k}} a^{-2^{k}}=e
$$

И

$$
\left(y b_{1}\right)^{2^{k}}=y^{2^{k}} b_{1}^{2^{k}}=b^{2^{k}} b^{-2^{k}}=e .
$$

Так как $f$ - автоморфизм максимального периода и элементы $x a_{1}^{-1}$ и $y b_{1}^{-1}$ имеют равные порядки, то существует такое целое число $t$, что $f^{t}\left(x a_{1}^{-1}\right)=y b_{1}^{-1}$. Но тогда

$$
\varphi^{t}(x H)=y H,
$$

т.е. $\varphi$ действительно является автоморфизмом максимального порядка группы $G / H$. Наконец, из существования изоморфизма $G / H \simeq K$ следует, что группа $K$ обладает автоморфизмами максимального порядка. Теперь доказательство теоремы следует из леммы 1.

\section{3. Группы порядка $2 p^{m}$}

Рассмотрим сначала группы порядка $2 p^{m}$. Как следует из теоремы 3 , для таких групп возможны только два варианта, а именно $C_{2} \ltimes C_{p^{m}}$ и $C_{2} \ltimes C_{p}^{m}$. Описание групп типа $C_{2} \ltimes C_{p^{m}}$, обладающих автоморфизмами максимального периода, было получено в работе [1], где доказано следующее утверждение.

Лемма 2. Пусть $G$ - группа с автоморфизмом максимального периода, причем $|G|=2 p^{m}$ и силовская р-подгруппа группы $G$ ииклическая. Тогда $G \simeq C_{2} \times C_{p^{m}}$.

Рассмотрим группы второго типа.

Лемма 3. Пусть $G$ - группа с автоморфизмом $f$ максимального периода, причем $G \simeq C_{2} \ltimes C_{p}^{m}$. Тогда $G \simeq C_{2} \times C_{p}^{m}$. 
Доказательство. Так как $G \simeq C_{2} \ltimes C_{p}^{m}$, то любой элемент группы $G$ можно однозначно представить в виде $a x$, где $a \in C_{2}$, а $x \in C_{p}^{m}$.

Пусть $a$ - элемент второго порядка и пусть $f(a)=a x$, где $x \in C_{p}^{m}$ - некоторый элемент порядка $p$. Тогда $|a x|=2$, и из равенства

$$
(a x)^{2}=(a x)(a x)=e
$$

следует, что

$$
a x a^{-1}=x^{-1} .
$$

Далее, если $f(x)=y$, то

$$
f^{2}(a)=f(a x)=a x y,
$$

причем $a x y$ также является элементом второго порядка. Отсюда, с учетом равенства $a x a^{-1}=x^{-1}$, сразу получаем

$$
a(x y) a^{-1}=(x y)^{-1}=x^{-1} y^{-1}=x^{-1}\left(a y a^{-1}\right),
$$

откуда

$$
\text { aya } a^{-1}=y^{-1} .
$$

При этом элемент ау является элементом второго порядка. Перебирая последовательно степени автоморфизма $f$, мы получим, что для любого элемента $z \in C_{p}^{m}$ выполняется равенство $a z a^{-1}=z^{-1}$ и элемент $a z$ является элементом второго порядка.

Таким образом, группа $G$ содержит один элемент порядка $1, p^{m}-1$ элементов порядка $p$ и $p^{n}$ элементов порядка 2 (это сам элемент $a$ и $p^{m}-1$ элементов вида $a x$, где $\left.x \in C_{p}^{m}\right)$.

Положим $N=p^{m}$; пусть $x_{1}=x, x_{2}, \ldots, x_{N-1}$ - все элементы порядка $p$, причем $f\left(x_{i}\right)=x_{i+1}$ для $i=1,2, \ldots, N-2$ и $f\left(x_{N-1}\right)=x_{1}$. Рассмотрим последовательность

$$
\begin{aligned}
a & \\
f(a) & =a x_{1} \\
f^{2}(a) & =f(a) f\left(x_{1}\right)=a x_{1} x_{2}, \\
f^{3}(a) & =f(a) f\left(x_{1}\right) f\left(x_{2}\right)=a x_{1} x_{2} x_{3}, \\
\ldots & \\
f^{N}(a) & =a=a x_{1} x_{2} \ldots x_{N-1} x_{1} .
\end{aligned}
$$

Отсюда получаем

$$
\left(x_{1} x_{2} \ldots x_{N-1}\right) x_{1}=e,
$$

но так как в произведение, стоящее слева в скобках, входят все элементы группы $G$ порядка $p$ по одному разу, то это произведение равно единице, то есть $x_{1}=e$, что противоречит нашему предположению.

Это означает, что группа $G$ содержит единственный элемент второго порядка, а именно, элемент $a$. Но тогда $x a x^{-1}=a$ для любого $x \in C_{p}^{m}$, то есть $a x=x a$ и группа $G$ абелева, что и завершает доказательство.

Из лемм 2 и 3 сразу следует теорема, дающая полное описание всех групп порядка $2 p^{m}$, обладающих автоморфизмами максимального периода.

Теорема 4. Пусть $G$ - группа порядка $2 p^{m}$, обладающая автоморфизмом максимального периода. Тогда $G$ - абелева группа одного из следующих двух типов:

1) $C_{2} \times C_{p}^{m}$,

2) $C_{2} \times C_{p^{m}}$. 


\section{4. Группы порядка $4 p^{m}$ с циклической силовской 2-подгруппой}

Теперь рассмотрим группы порядка $4 p^{m}$, у которых силовская 2-подгруппа циклическая, то есть группы типов $C_{4} \ltimes C_{p^{m}}$ и $C_{4} \ltimes C_{p}^{m}$ для некоторого простого $p>2$. Начнем со следующего утверждения.

Лемма 4. Пусть $G$ - группа типа $C_{4} \ltimes C_{p^{m}}$ или $C_{4} \ltimes C_{p}^{m}$, обладающая автоморфизмом $f$ максимального периода. Тогда группа $G$ абелева.

Доказательство. Пусть $C_{4}=\langle a\rangle$. Покажем, что если $b$ - любой элемент из силовской $p$-подгруппы группы $G$, то выполняется равенство $a b^{2}=b^{2} a$, откуда и будет следовать доказываемое утверждение.

Отметим, что $a^{-1} \neq a$ и, так как $f$ - автоморфизм максимального периода, то существуют такие целые числа $n$ и $m$, что

$$
f^{n}(a)=a^{-1} \text { и } f^{m}(b)=b^{-1} .
$$

Пусть $d=[n, m]$ - наименьшее общее кратное чисел $n$ и $m$. Тогда если $d=n d_{1}=$ $m d_{2}$, то числа $d_{1}$ и $d_{2}$ не могут быть четными одновременно. Более того, $d_{2}$ всегда является нечетным. В самом деле, число силовских 2-подгрупп в группе $G$ равно $p^{t}$ для некоторого $t \leqslant m$, то есть группа $G$ содержит $2 p^{t}$ элементов четвертого порядка (образующие соответствующих силовских подгрупп). Это означает, что $n=p^{t}$, а тогда $d_{2}$ нечетно. Поэтому рассмотрим два варианта.

1) Пусть $d_{1}$ - нечетное, $d_{2}$ - нечетное. Тогда, так как $|a|=\left|b a b^{-1}\right|$, то

$$
b a^{-1} b^{-1}=f^{d}\left(b a b^{-1}\right)=b^{-1} a^{-1} b,
$$

откуда следует, что $b^{2} a=a b^{2}$. Так как $(|b|, 2)=1$, то отсюда следует, что $b a=a b$ и, в частности, $a^{2} b=b a^{2}$.

2) Пусть $d_{1}$ - четное и $d_{2}$ - нечетное. Тогда, так как $|a|=\left|b a b^{-1}\right|$, то

$$
b a b^{-1}=f^{d}\left(b a b^{-1}\right)=b^{-1} a b,
$$

откуда следует, что $b^{2} a=a b^{2}$. Отсюда, как и выше, получаем, что $b a=a b$.

Таким образом, во всех возможных вариантах оказывается, что $a b=b a$, то есть группа $G$ абелева.

Следствие 2. Группы порядка $4 p^{m}$ с ииклической силовской 2-подгруппой не имеют автоморфизмов максимального периода.

Доказательство. В самом деле, группа, удовлетворяющая условиям, является абелевой, а все абелевы группы, обладающие автоморфизмами максимального периода, описаны в теореме 3 .

\section{5. Группы порядка $2^{n} p^{m}$}

Рассмотрим теперь группы порядка $2^{n} p^{m}$, у которых силовская 2-подгруппа является элементарной абелевой 2-группой, то есть группы типов $C_{2}^{n} \ltimes C_{p^{m}}$ и $C_{2}^{n} \ltimes C_{p}^{m}$. Предложение 1. Пусть $G$ - группа типа $C_{2}^{n} \ltimes C_{p^{m}}$ или $C_{2}^{n} \ltimes C_{p}^{m}$. Тогда она содержит нечетное число элементов второго порядка. 
Доказательство. В самом деле, любой элемент группы $G$ однозначно представляется в виде $a b$, где $a \in C_{2}^{n}$ и $b$ - элемент из силовской $p$-подгруппы. При этом, если элемент $a b$ имеет порядок 2 , то есть если $(a b)^{2}=e$, то $a b a^{-1}=b^{-1}$ (так как $a^{2}=e$, то $\left.a=a^{-1}\right)$. При этом для любого целого числа $k$ выполняется равенство $a b^{k} a^{-1}=b^{-k}$, то есть элемент $a b^{k}$ также является элементом второго порядка. Так как $b$ порождает подгруппу порядка $p^{t}$ при некотором $t \leqslant m$, то эта подгруппа содержит четное число неединичных элементов. Следовательно, для фиксированного элемента $a$ второго порядка число элементов группы $G$ вида $a b^{k}$ четно.

Подгруппа $C_{2}^{n}$ содержит $2^{n}-1$ элементов второго порядка. При этом каждому элементу $a \in C_{2}^{n}$ соответствует четное число (возможно, нуль) элементов вида $a b$. Следовательно, всего группа $G$ содержит нечетное число элементов второго порядка.

Теперь мы можем доказать следующую теорему, завершающую классификацию конечных групп, обладающих автоморфизмами максимального периода.

Теорема 5. Группа $G$ типа $C_{2}^{n} \ltimes C_{p^{m}}$ или $C_{2}^{n} \ltimes C_{p}^{m}$, обладающая автоморфизмом максимального периода, абелева.

Доказательство. Действительно, пусть $a \in C_{2}^{n}$ и $b-$ элемент силовской p-подгруппы. Так как $f$ - автоморфизм максимального периода, то существуют такие целые числа $s$ и $t$, что

$$
f^{s}(a)=a \text { и } f^{t}(b)=b^{-1},
$$

причем $s$ нечетно, так как $G$ содержит нечетное число элементов второго порядка. Пусть $d=[s, t]$ - наименьшее общее кратное чисел $s$ и $t$. Тогда если $d=s d_{1}=t d_{2}$, то $d_{2}$ является нечетным. Рассмотрим два варианта.

1) Пусть $d_{1}$ - нечетное, $d_{2}$ - нечетное. Тогда, так как $|a|=\left|b a b^{-1}\right|$, то

$$
b a b^{-1}=f^{d}\left(b a b^{-1}\right)=b^{-1} a b,
$$

откуда следует, что $b^{2} a=a b^{2}$. Так как $(|b|, 2)=1$, то отсюда следует, что $b a=a b$.

2) Пусть $d_{1}$ - четное и $d_{2}$ - нечетное. Тогда, так как $|a|=\left|b a b^{-1}\right|$, то

$$
b a b^{-1}=f^{d}\left(b a b^{-1}\right)=b^{-1} a b,
$$

откуда следует, что $b^{2} a=a b^{2}$. Отсюда, как и выше, получаем, что $b a=a b$.

Таким образом, во всех возможных вариантах оказывается, что $a b=b a$, то есть группа $G$ абелева.

Собирая полученные результаты,сформулируем теорему, дающую полное описание всех конечных групп, обладающих автоморфизмами максимального периода.

Теорема 6. Пусть $G$ - конечная группа, обладающая автоморфизмами максимального периода. Тогда $G$ изоморфна одной из следующих групп:

1) $C_{p}^{n}, p$ - простое число,

2) $C_{4}$

3) $C_{p^{n}}, p$ - простое число, $p \neq 2$,

4) $C_{2}^{n} \times C_{p}^{m}, p$ - простое число, $p \neq 2 u\left(2^{n}-1, p^{m}-1\right)=1$,

5) $C_{2}^{n} \times C_{p^{m}}, p-$ простое число, $p \neq 2$ u $\left(2^{n}-1, p^{m-1}(p-1)\right)=1$. 


\section{Список литературы}

1. Акишин А.В., "Группы с автоморфизмами, порождающими рекуррентные последовательности максимального периода", Дискретная математика, 26:1 (2014), 3-9.

2. Глухов М.М., Ларин С.В., “Абелевы и метабелевы группы с регулярными автоморфизмами и полуавтоморфизмами", Математические заметки, 1:2 (1972), 727-738.

3. Кузьмин А.С., Куракин В.Л., Нечаев А.А., "Псевдослучайные и полилинейные последовательности", Труды по дискретной математике, 1 (1997), 139-202.

4. Кузьмин А.С., Маршалко Г.Б., Нечаев А.А., "Восстановление линейной рекурренты над примарным кольцом вычетов по ее усложнению", Математические вопросы криптографии, 1:2 (2010), 31-56.

5. Кузьмин А.С., Нечаев А.А., “Линейные рекуррентные последовательности над кольцами Галуа", Успехи математических наук, 48:1 (1993), 167-168.

6. Лидл Р., Нидеррайтер Г., Конечные поля, том 2, М.: "Мир", 1988.

7. Макаренко М.Ю., Хухро Е.И., "Конечные группы с почти регулярным автоморфизмом порядка четыре", Алгебра и логика, 45:5 (2006), 575-602.

8. Нечаев А.А., “Линейные рекуррентные последовательности над коммутативными кольцами", Дискретная математика, 3:4 (1991), 105-127.

9. Babbage S., Dodd M., "The MICKEY stream ciphers:", In: The eSTREAM Finalists, Springer, 2008, 191-209.

10. Berbain C., Billet O., Canteaut A., Courtois N., Debraize B., Gilbert H., Goubin L., Gouget A., Granboulan L., Lauradoux C., Minier M., Pornin T., Siber H., "DECIM v2", In: The eSTREAM Finalists, Springer, 2008, 140-151.

11. De Cannière C. "Trivium: A stream cipher construction inspired by block cipher design principles", ISC'2006, Lect. Notes Comput. Sci., 4176 (2006), 171-186.

12. Geffe R.R., "How to protect data with ciphers that really hard to break", Electronics, 64:1 (1973), 99-101.

13. Günther C.G., "Alternating step generators controlled by de Bruijn sequences", EUROCRYPT'87, Lect. Notes Comput. Sci., 304 (1988), 5-14.

14. Jennings S.M., "Autocorrelation function of the multiplexed sequence", IKE Proceedings, 131:2 (1984), 169-172. 